\title{
Neural substrates of rumination tendency in non-depressed individuals
}

\author{
Camille Piguet $^{\mathrm{a}, *}$, Martin Desseilles ${ }^{\mathrm{a}, \mathrm{b}, \mathrm{c}}$, Virginie Sterpenich ${ }^{\mathrm{a}}$, Yann Cojan ${ }^{\mathrm{a}}$, \\ Gilles Bertschy ${ }^{\mathrm{d}}$, Patrik Vuilleumier ${ }^{\mathrm{a}, \mathrm{e}}$ \\ a Department of Neuroscience, Faculty of Medicine, University of Geneva, Switzerland \\ ${ }^{\mathrm{b}}$ Cyclotron Research Center, University of Liège, Belgium \\ ${ }^{\mathrm{c}}$ Department of Psychiatry, Geneva University Hospital, Switzerland \\ d Department of Psychiatry and Mental Health, Strasbourg University Hospital, University of Strasbourg, INSERMu1114, France \\ e Department of Clinical Neuroscience, Geneva University Hospital, Switzerland
}

\section{A R T I C L E I N F O}

\section{Article history:}

Received 7 April 2014

Accepted 10 September 2014

Available online 19 September 2014

\section{Keywords:}

fMRI

Depression

Rumination

Resting state

RRS

\begin{abstract}
A B S T R A C T
The tendency to ruminate, experienced by both healthy individuals and depressed patients, can be quantified by the Ruminative Response Scale (RRS). We hypothesized that brain activity associated with rumination tendency might not only occur at rest but also persist to some degree during a cognitive task. We correlated RRS with whole-brain fMRI data of 20 healthy subjects during rest and during a face categorization task with different levels of cognitive demands (easy or difficult conditions). Our results reveal that the more subjects tend to ruminate, the more they activate the left entorhinal region, both at rest and during the easy task condition, under low attentional demands. Conversely, lower tendency to ruminate correlates with greater activation of visual cortex during rest and activation of insula during the easy task condition. These results indicate a particular neural marker of the tendency to ruminate, corresponding to increased spontaneous activity in memory-related areas, presumably reflecting more internally driven trains of thoughts even during a concomitant task. Conversely, people who are not prone to ruminate show more externally driven activity.
\end{abstract}

(c) 2014 Elsevier B.V. All rights reserved.

\section{Introduction}

A trait feature of different psychiatric disorders is negative repetitive thinking (Watkins, 2008), and in particular rumination. According to the most frequent definition of this phenomenon, "ruminations are repetitive and passive thinking about symptoms of depression and the possible causes and consequences of these symptoms" (Nolen-Hoeksema, 1991). Although rumination in general can be conceptualized as a form of thinking found in different pathologies and present in everybody to some degree (NolenHoeksema \& Watkins, 2011; Wells, 2004), it is most often related to depressive mood (Nolen-Hoeksema, 2000) and has consistently been linked to negative affect (Thomsen, 2006). In this perspective, depressive rumination is seen as a particular response style

\footnotetext{
* Corresponding author at: Laboratory for Neurology and Imaging of Cognition, Neuroscience Department, Faculty of Medicine, University of Geneva, University Medical Centre, 1 rue Michel Servet, 1211 Genève 4, Switzerland. Tel.: +4122379 5324; fax: +41223795402.

E-mail address: camille.piguet@unige.ch (C. Piguet).
}

that, instead of being oriented to problem solving, tends to enhance internal focus, pessimism, and perseverative cognitions, which may in turn exacerbate low mood (Watkins, 2008; Nolen-Hoeksema, 1991; Nolen-Hoeksema, Wisco, \& Lyubomirsky, 2008; Spasojevic \& Alloy, 2001). Thus, accumulating evidence points toward rumination being an important vulnerability factor in the development of depression (McLaughlin \& Nolen-Hoeksema, 2011; Wiersma et al., 2011) and constituting a maladaptive mental habit (Watkins \& Nolen-Hoeksema, 2014). The tendency to ruminate is commonly measured by the Ruminative Response Scale (RRS) (Davis \& NolenHoeksema, 2000; Nolen-Hoeksema et al., 2008; Ray et al., 2005; Spasojevic \& Alloy, 2001; Thomas et al., 2011; Whitmer \& Banich, 2007).

Depression is also associated with different kinds of cognitive dysfunctions (for a review, see Gotlib \& Joormann, 2010). A few specific deficits have been linked with ruminations, particularly cognitive inflexibility and difficulties in disengaging attention from irrelevant information (Joormann \& D'Avanzato, 2010; Koster, De Lissnyder, Derakshan, \& De Raedt, 2011; Whitmer \& Banich, 2007). This might be even stronger for negative material (Lissnyder, Koster, Derakshan, \& De Raedt, 2010; Koster, De Lissnyder, \& De 
Raedt, 2013). However, the link between rumination and allocation of attention is still unclear, because the direction of the causality is difficult to demonstrate. On the one hand, a general impairment in cognitive resources or control could explain the difficulties of the subjects to allocate their attention and thoughts away from intrusive material (Koster et al., 2011; Levens, Muhtadie, \& Gotlib, 2009). On the other hand, the dominance of ruminations in the content of attention could explain the difficulties in allocating or shifting cognitive resources to other thoughts (Philippot \& Brutoux, 2008). Furthermore, a state of rumination might potentially result from deficient cognitive control normally allowing the inhibition of irrelevant thoughts and/or from increased generation of intrusive material through spontaneous associations and elaborative emotional processing (Mandell, Siegle, Shutt, Feldmiller, \& Thase, 2014; Piguet et al., 2010). Hence, the functional relationships of rumination tendency with attention control remain to be better understood (for a recent review, see Whitmer \& Gotlib, 2013).

The neural substrates of rumination remain largely unresolved. Recent studies point to an involvement of heightened emotional reactivity, but also weaker cognitive control. An early study (Ray et al., 2005) examined how brain activation during cognitive reappraisal of emotional pictures varied with trait rumination tendency, and found that the latter correlated with higher amygdala response when increasing negative affect, and lower medial prefrontal activity when decreasing negative affect. Another study (Johnson, Nolen-Hoeksema, Mitchell, \& Levin, 2009) reported that higher trait rumination in depressed individuals correlated with greater difficulties in deactivating the posterior cingulate cortex (PCC), a region associated with self-focused processes, when engaged in an external (non-self-referential) task. They also reported lower activation of anterior cingulate cortex (ACC). Cooney, Joormann, Eugene, Dennis, and Gotlib (2010), using a rumination induction task, found that depressed patients showed increased activation in amygdala, rostral anterior cingulate cortex/medial prefrontal cortex (PFC), dorsolateral PFC, and parahippocampal cortex, during rumination relative to an abstract distraction task. Another study, using the recall of autobiographical negative memories and subsequent focus on elicited emotions as a proxy for rumination, found that the latter correlated with activity in subgenual ACC (sgACC) and medial PFC (Kross, Davidson, Weber, \& Ochsner, 2009). More recently, Freton et al. (2013) showed that lower brooding scores measured by the RRS (emphasizing the maladaptive component of self-reflection) correlated with increased activation of the posterior midline structures during analytical compared to experiential self-focus, which may account for impaired cognitive control on self-focus in high brooders. Finally, in depressed patients, using a factor structure derived from multiple questionnaires and a paradigm alternating cognitive and emotional tasks in depressed patients, Mandell et al. (2014) showed that trait rumination correlated not only with amygdala but also hippocampus activity. Other regions in PCC, MPFC, dorsolateral PFC, and anterior insula also exhibited differential patterns as a function of ruminations traits. Taken together, these studies suggest that self-referential and memory-related activity, in addition to emotional factors, may be associated with the presence and content of ruminative thinking, but they do not elucidate a possible role for attentional control abilities in promoting the appearance or persistence of rumination activity.

A few other recent fMRI studies investigated the link between depressive rumination and brain activity in the so-called default mode network (DMN), which is typically associated with selfreflective processes and observed in resting state conditions, but deactivated during attention demanding tasks. Hyperconnectivity between components of the DMN in PCC and sgACC was found at rest for patients with Major Depressive Disorder (MDD), correlating with RRS scores, and more specifically the brooding subscore
(Berman, Peltier, et al., 2011). Another study comparing DMN and task-positive networks in MDD showed that ruminations were associated with increased DMN dominance at rest (Hamilton et al., 2011). Finally, by computing whole-brain correlation between RRS and resting state activity, Kühn, Vanderhasselt, De Raedt, and Gallinat (2012) found a negative relation with right inferior frontal gyrus, right ACC, and sgACC. They also found increased functional connectivity of the left striatum with left inferior frontal gyrus in healthy individuals experiencing more unwanted thoughts (Kühn, Vanderhasselt, De Raedt, \& Gallinat, 2013). This is consistent with higher rumination scores (as assessed by the RRS) being associated at rest with lower connectivity between sgACC and the middle and inferior frontal gyri in adolescents with a first-episode depression (Connolly et al., 2013). Again, increased activity and/or connectivity within areas of the DMN involved in emotional and self-referential processing might contribute to ruminations tendency, in addition to decreased activity in cognitive control regions. Altogether, this literature points to a link between rumination and cortical midline structure (Nejad, Fossati, \& Lemogne, 2013), with an imbalance between the recruitment of externally directed attention/executive control networks (in fronto-parietal cortices) and internally directed self-referential/memory networks (in midline and limbic brain systems), particularly during rest (Marchetti, Koster, Sonuga-Barke, \& De Raedt, 2012). However the relationships between the attention state, the cognitive control abilities and the activity in other brain structures remain unclear in the context of rumination.

To clarify the neural substrates of rumination tendencies in the absence of depressive illness, the current study used fMRI in the same group of healthy participants, both during a demanding cognitive task with different degrees of attentional load, and during a resting state condition with no external demands. First, we specifically tested the hypothesis that lower attentional shifting abilities in high ruminators may release ruminative processes and thus lead to higher activation of self-related regions during lower cognitive load. Second, we hypothesized that a direct comparison between the resting and cognitive states, hence two separate datasets from the same population, should allow us to determine whether the neural activation pattern associated with rumination tendency seen at rest would resemble the pattern seen in the low demanding condition of the cognitive task. Specifically, we expected not only that brain areas reflecting attention control should show reduced engagement in people with higher rumination tendencies, but also that this should in turn promote a release of activity in brain areas involved in the generation of ruminative thoughts.

\section{Methods}

\subsection{Participants}

Twenty healthy subjects ( 10 women and 10 men) participated in this study recruited through local advertisement, and filled informed consent. Participants reported having no neurologic or psychiatric medical history and taking no medication. Mean age was 24.9 (std 5.457, ranging from 18 to 37). The Ethical Committee for Psychiatry and Rehabilitation of the University Hospital of Geneva approved this study.

\subsection{Questionnaires}

Participants filled the 22-items of the Ruminative Response Scale (RRS) (NolenHoeksema \& Morrow, 1991) on the day of the MRI session. This questionnaire assesses the tendency of individuals to ruminate when they feel depressed. This trait measure has been widely used in clinical studies (e.g. Vanderhasselt \& De Raedt, 2012) as well as neuroimaging studies (see Section 1). Participants also filled the Beck Depression Inventory-II (BDI, Beck, Steer, \& Brown, 1996) and the Beck Anxiety Inventory (BAI, Beck, Epstein, Brown, \& Steer, 1988).

\subsection{Behavioral task}

As an active cognitive condition, we used a task switching paradigm (Piguet et al., 2013) with emotional faces. On each trial, participants first saw a cue for $150 \mathrm{~ms}$, either the word "emotion" or "color" or "gender", instructing them to judge 
the upcoming face pictures along this particular dimension. The cue stayed on the screen while three faces appeared (arranged in a triangle) that were either male or female, sad or happy, and colored in red or green. Participants had to decide (as rapidly as possible) which among the three faces was different of the two others according to the preceding instruction cue. They answered with a button box with a direct stimulus-mapping rule: button 1 for left face, button 2 for middle face, and button 3 for right face. The cue and face stimuli stayed on the screen until the answer. There were 24 different faces taken from the Karolinska Directed Emotion Face database (Lundqvist, Flykt, \& Ohman, 1998). Participants did 4 sessions of 216 trials each (see Piguet et al., 2013 for additional details on the task design).

As in other task switching paradigms (De Lissnyder, Koster, Derakshan, \& De Raedt, 2010; Mayr \& Keele, 2000), each trial was defined in terms of its position in the task sequence. Conditions with a higher cognitive load were those where the participant has to switch from one task to another (e.g., Color-Gender-Emotion, or Gender-Emotion-Gender), compared to situations involving the same task (e.g., Emotion-Emotion-Emotion). Sequences where participants have to switch twice consecutively are considered to require overcoming an additional inhibition component when returning to the same task (Color-Gender-Color), relative to two successive switch without returning (Color-Gender-Emotion). Altogether, these different sequences and task conditions yielded 12 experimental conditions (RepetitionEmotion, RepetitionGender, RepetitionColor, SwitchEmotion, SwitchGender, SwitchColor, DoubleswitchEmotion, DoubleswitchGender, DoubleswitchColor, InhibitionEmotion, InhibitionGender, InhibitionColor); but for the purpose of our study, we mainly focused on the contrast between the easy condition (Repetition) and the more difficult conditions requiring switching (Switch, Doubleswitch, Inhibition), Color, Gender and Emotion tasks pooled together. This resulted in slightly more switch trials than repetition trials (respectively, 384 repetitions trials and 480 switch trials for each participant).

For the resting state condition, participants were simply asked to stay relaxed for $10 \mathrm{~min}$, closing their eyes without falling asleep, and letting their thoughts wander. The screen was turned off. Immediately after the scanning session, visual scales were proposed to the participant to rate their level of sleepiness and anxiety.

\section{4. fMRI data acquisition}

Data were acquired with a $3 \mathrm{~T}$ magnetic resonance (MR) scanner (TIM Trio, Siemens) using a gradient echo-planar (EPI) sequence [35 transverse slices with $20 \%$ gap, voxel size: $3 \mathrm{~mm} \times 3 \mathrm{~mm} \times 3.6 \mathrm{~mm}$, repetition time (TR): $2040 \mathrm{~ms}$, echo time (TE): $30 \mathrm{~ms}$, flip angle (FA): $80^{\circ}$, field of view (FOV): $192 \mathrm{~mm}$ ]. For the cognitive task, between 193 and 318 scans (mean of 260) were acquired for each run of the task, depending on how fast the participant answered. Three runs were acquired for each subject. The resting state session comprised 500 scans [EPI, matrix size $64 \times 64 \times 21$, voxel size $3.75 \times 3.75 \times 5.25$, TR $1100 \mathrm{~ms}$, slice thickness 4.2 with 1.05 gap]. A structural MR scan was also acquired at the end of the fMRI session [T1weighted 3D MP-RAGE sequence, TR: $1900 \mathrm{~ms}$, TE: $2.32 \mathrm{~ms}$, TI: $900 \mathrm{~ms}$, FA: $9^{\circ}$, FOV: $230 \mathrm{~mm}$, matrix size $256 \times 256 \times 192$, voxel size: $.898 \mathrm{~mm} \times .898 \mathrm{~mm} \times .9 \mathrm{~mm}$ ]. All acquisitions were obtained on the same day. Stimuli were displayed using an LCD projector (CP-SX1350, Hitachi, Japan) on a screen positioned at the rear of the scanner, which the participants could comfortably see through a mirror mounted on the standard 12-channel head coil.

\subsection{Data analysis}

fMRI data were analyzed using SPM5 (http://www.fil.ion.ucl.ac.uk) implemented in Matlab R2007b (Mathworks). All functional scans were realigned using iterative rigid body transformations that minimize the residual sum of square between the first and subsequent images. They were normalized to the MNI EPI template (2D spline, voxel size: $2 \mathrm{~mm} \times 2 \mathrm{~mm} \times 2 \mathrm{~mm}$ ) and spatially smoothed with a Gaussian kernel with full-width at half maximum (FWHM) of $8 \mathrm{~mm}$. A highresolution structural image was co-registered with the mean image of the EPI series and normalized.

Data from the cognitive task were processed using a two-step analysis, taking into account the intraindividual and interindividual variance, as described elsewhere (Friston et al., 1994). Statistical analyses were carried out using the GLM for event-related design as implemented in SPM5. The onsets of conditions of interest were convolved with the canonical hemodynamic response function (HRF) and used as a regressor in the individual design matrix (first level). The individual statistical images from each condition were used in a second-level ANOVA analysis to create the contrasts of interest, i.e. Repetition (easy) versus Switching (difficult) conditions.

The resting state data was treated with the same pre-processing steps, except for $3 \mathrm{~mm} \times 3 \mathrm{~mm} \times 3 \mathrm{~mm}$ normalization. They were subsequently analyzed with independent component analysis (ICA) using the GIFT toolbox (Calhoun, Adali, Pearlson, \& Pekar, 2001; Schöpf, Windischberger, Kasess, Lanzenberger, \& Moser, 2010). This data-driven method allows the creation of distinct maps of brain regions that show the same temporal pattern of activity. The group maps were then inspected to select networks of interest for subsequent analyses.

Finally, contrasts from the second level analysis of the cognitive task and maps from the ICA analysis of resting state were correlated independently in SPM, using a multiple linear regression model with the scores of each individual from the RRS,
BDI, and BAI. Although some of these measures were correlated (BDI, BAI) or tended to correlate (RRS, BDI), we chose to use a multiple regression in order to estimate correlations with the most complete model and thus account for all sources of variance. The correlations with ICA components were selectively searched within significant regions of the ICA map by using an inclusive mask (thresholded at $p=.05$ ). Correlation results are reported at a statistical threshold of $p<.001$ uncorrected at the voxel peak.

Note that here we did not intend to compare switching performance between emotional and non-emotional tasks in our cognitive tasks, as this has been reported elsewhere (see Piguet et al., 2013) and this factor did not constitute an emotion induction procedure relevant to RRS (which is an individual trait dimension). Instead we primarily focused on (1) the tendency to ruminate in relation to attention load, and (2) the comparison of rumination activity at rest with the different attention load condition.

\section{Results}

\subsection{Population}

Our participants had a mean RRS score of 40.5 (min 24, max 65 , std 9.03). There are no norms for the general population, but a clinical study with different patient categories reported a mean score of 65 (std 11 ) for currently depressed patients, 47.7 (std 14.5 ) for remitted patients, and 36.5 (std 8.2) for never depressed participants (Watkins \& Baracaia, 2002). The mean score on the Beck Depression Inventory was 4.75 ( $\min 0, \max 19$, std 4.72 ). Rumination and depression scores were not correlated $(r(20)=.278$, $p=.117)$. The mean score on the Beck Anxiety Inventory was 4.2 (min 0, max 15, std 3.98).

\subsection{Behavioral results}

In the cognitive task, reaction times (RT) in the easy condition (repetition) were compared with those in the three difficult conditions (switching trials) pooled together. The mean RT for repetition trials was $1127 \mathrm{~ms}(\mathrm{std}=31.9)$ and $1189 \mathrm{~ms}(\mathrm{std}=42.1)$ for switch trials. These two conditions were significantly different (paired $t$ test, $t=4.066, p=.001$ ), confirming the existence of a switching cost (Meiran, 2010). This difference was also found when comparing each switch condition individually with repetition (all $p<.005$ ). Reaction times and switch cost did not correlate with RRS, but accuracy across all trials showed a negative correlation with RRS $(r(20)=-.54, p=.011)$.

\section{3. fMRI results}

The ICA analysis of resting state fMRI data identified 36 distinct maps. Out of these, we selected two representative maps known to be associated with cognitive networks comparable with the taskrelated effects, i.e. an attentional network map involving large, bilateral and symmetric activations in the intraparietal cortex (IPS), and a visual network map involving large, bilateral, and symmetric activations in the occipito-temporal cortex.

By entering the RRS score in a linear regression analysis of each map in SPM, we observed a significant positive correlation for the visual network map between resting activity in the entorhinal cortex and the score of rumination (Fig. 1A). Conversely, we observed a negative correlation for the attention network map between rumination scores and activity in the left middle occipital gyrus (see Table 1 and Fig. 2A). This suggests that the more the subjects tend to ruminate, the more they activate memory-related areas, but the less they recruit extrastriate visual areas during the resting session.

Two other ICA maps overlapped with the classical DMN (Buckner, 2012), but with a predominance of medial prefrontal activations in one component and of medial parietal activations in the other. The first frontal-dominant DMN map showed a negative correlation of RRS with ACC and PCC (both $p<.001$ uncorrected), whereas the second map also showed a selective negative 
Table 1

Correlations between Ruminative Response Scale (RRS) and anatomical regions; ( $p<.001$ unc. except * $p<.005)$.

\begin{tabular}{|c|c|c|c|c|c|}
\hline MNI coordinates & $x$ & $y$ & $z$ & Voxels & $Z$ score \\
\hline \multicolumn{6}{|c|}{ Resting state, visual map, RRS positive } \\
\hline Left entorhinal cortex & -16 & -20 & -28 & 11 & 3.81 \\
\hline \multicolumn{6}{|c|}{ Resting state, attentional map, RRS negative } \\
\hline Left medial occipital gyrus (visual) & -24 & -96 & 0 & 10 & 3.99 \\
\hline \multicolumn{6}{|c|}{ Cognitive task, easy vs. difficult conditions, RRS positive } \\
\hline Left entorhinal cortex & -15 & -18 & -24 & 3 & 4.08 \\
\hline \multicolumn{6}{|c|}{ Cognitive task, easy vs. difficult conditions, RRS negative } \\
\hline Right insula & 42 & 18 & -6 & 10 & 3.8 \\
\hline
\end{tabular}

correlation in PCC (but slightly weaker, at $p=.005$ uncorrected) and no effect in ACC.

For the cognitive task, our whole-brain analysis focused on the main conditions of interest and correlations with RRS (more detailed results concerning all conditions are reported elsewhere; see Piguet et al., 2013). We first computed the main effect of condition by contrasting easy versus difficult trials. As expected, the difficult condition (contrast Switch $>$ Repetition) produced significant increases in regions associated with attentional shifting and monitoring, including bilateral superior parietal lobules (SPL) and PCC, replicating previous work with similar tasks (Chiu \& Yantis, 2009; Kim, Cilles, Johnson, \& Gold, 2012; Philipp, Weidner, Koch, \& Fink, 2012; Piguet et al., 2013). These activations are consistent with the predicted increase in attentional demands on switch trials and a recruitment of brain areas typically associated with cognitive control and flexibility. Conversely, the easy condition (contrast Repetition $>$ Switch) produced significant activations in the left caudate nucleus, the right inferior frontal gyrus, the left superior frontal gyrus, and the right dorsomedial PFC. This network is compatible with brain areas engaged in learning and maintaining stimulus-response associations (Boettiger \& D'Esposito, 2005; Brass \& von Cramon, 2004), and suggests that subjects recruited more "procedural" and automatized sensorimotor processes in this condition.

More importantly, we then performed a whole-brain regression analysis with rumination scores from each individual, using the contrast "easy > difficult", which should highlight patterns of brain activity under low attentional demand, more similar to the resting state. Results showed a significant positive correlation between
A.
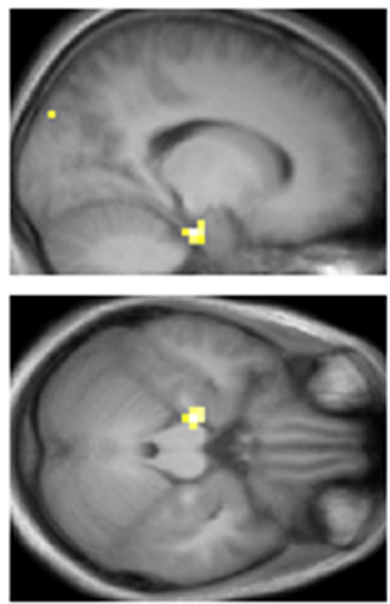

B.
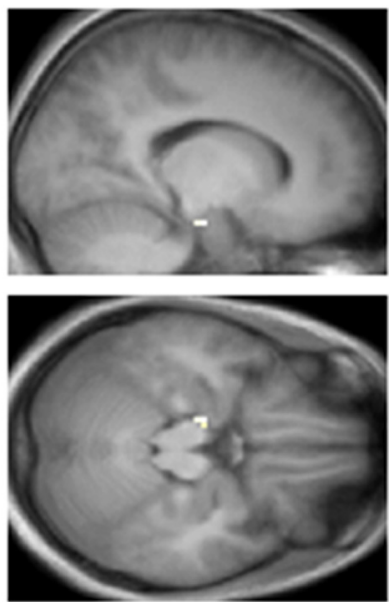

Fig. 1. Positive correlation with Ruminative Response Scale (RRS). (A) Correlation between high RRS and entorhinal cortex in the visual map of the resting state; (B) correlation between high RRS and entorhinal cortex in the easy condition of the cognitive task.
RRS and a medial temporal lobe region strikingly similar to that observed in the previous analysis of resting state: i.e. the left entorhinal cortex (see Fig. 1 B and Table 1). Fig. 3 plots the parameter estimates of activity in this region and the correlation with rumination scores. This relation held true $(p=.009)$ even when removing one extreme point (RRS score $=65$ ). Further inspection of this correlation for each task condition separately showed that this effect in entorhinal cortex was driven by both increases during the easy condition (Repetition) and decreases during the difficult condition (Switch). Finally, when looking at the opposite (negative) correlation, we found that higher RRS scores were associated with lower activity in the right anterior insula during the easy vs. difficult condition (Table 1 and Fig. 2B). No other correlations were found at the whole brain level.

When repeating the same whole-brain parametric analysis with brooding subscore (instead of total RRS) as a linear regressor, we found similar clusters in the entorhinal region during the easy condition of the attentional task (peak $x y z=-15,-18,-21$, $Z=5.25, p=.001 \mathrm{unc}$.) as well as during the resting state condition for the visual map from ICA although with a lower threshold (peak $x y z=-15,-24,-30, Z=2.45, p=.01$ unc.). Brooding scores also correlated negatively with activation in visual areas during rest for the attentional map $(x y z=-20,-100,-4, Z=2.68, p=.01$ unc.) but not with the insula during the easy condition. This weaker effect found for brooding during rest might reflect that ruminations at rest are also linked to more adaptive components of rumination, such as reflection and problem solving in association with mindwandering, whereas intrusive thoughts during low attention demands are more specifically linked to the maladaptive component associated with brooding.
A.
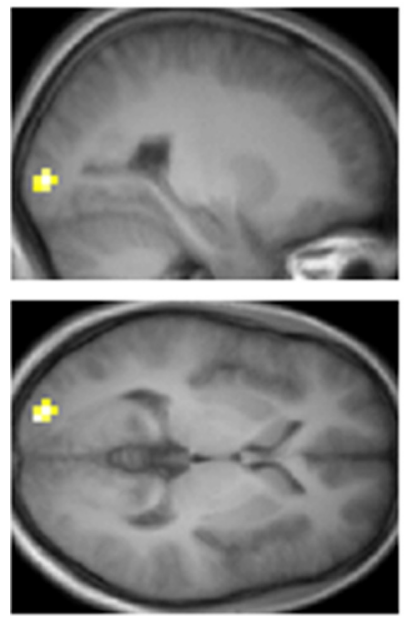

B.
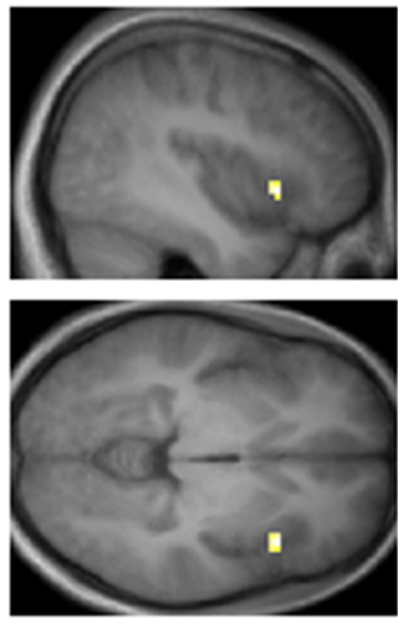

Fig. 2. Negative correlation with Ruminative Response Scale (RRS). (A) Correlation between low RRS and visual cortex; (B) correlation between low RRS and insula in the easy condition of cognitive task. 


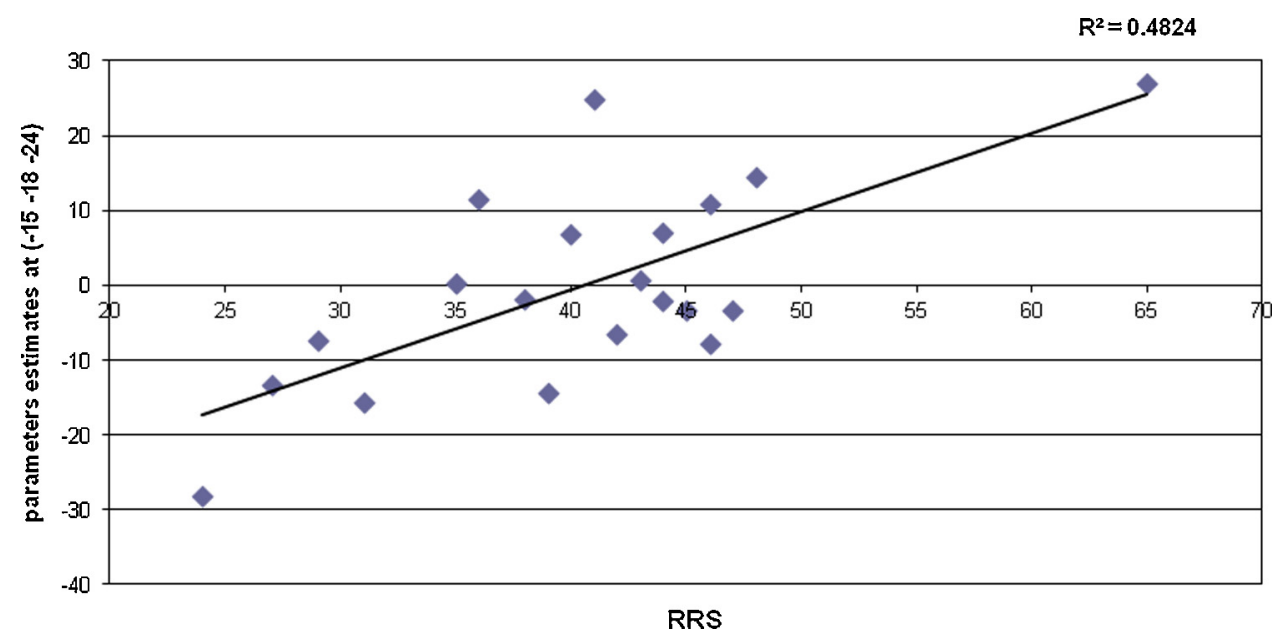

Fig. 3. Correlation between Ruminative Response Scale (RRS) and parameter estimates of entorhinal cortex in easy condition.

\section{Discussion}

We correlated scores on the Ruminative Response Scale (RRS) with brain activation patterns measured by fMRI, and compared these patterns during both an attention-demanding cognitive task (task-switching) and rest in the same participants. We found similar activation in the entorhinal cortex in both cases, which positively correlated with RRS scores during the resting state but also during the easy, less demanding condition of our cognitive task. This similarity of effects in the entorhinal cortex is all the more striking that it was observed in two totally separate datasets, corresponding to very different behavioral conditions. In addition, we also found that RRS scores correlated negatively with activity in extrastriate visual areas during rest, whereas they correlate negatively with the right anterior insula in the easy cognitive task condition.

These results provide novel support to the hypothesis that people with a propensity to ruminate, even when non-depressed, tend to recruit brain systems mediating the retrieval of personal memories and self-related information more strongly or persistently than non-ruminators. The entorhinal cortex is indeed intimately linked to autobiographical memories and familiarity (De VanssayMaigne et al., 2011). The medial temporal lobe in general, and more particularly the parahippocampal gyrus, has also been postulated to mediate self-referential associations in depression (Bar, 2009). Increased connectivity between this region and the posterior cingulate cortex could mediate the relationship between autobiographical memories and rumination and represent a neural substrate of vulnerability to depressive episodes (Zamoscik, Huffziger, Ebner-Priemer, Kuehner, \& Kirsch, 2014). A link between rumination tendency and hippocampus activation has also recently been suggested (Mandell et al., 2014). Our findings thus provides novel evidence that increased activity in this region may constitute a neural marker of rumination tendencies even in the absence of clinical depression, with spontaneous recruitment at rest as well as during an active task with low attentional demand.

By contrast, the reverse correlation observed in visual areas during rest suggests that individual with higher self-focus and ruminations allocate less resources to the processing of sensory visual inputs from the external world and/or engage less in visual imagery during rest. This converges with other findings that higher reactivity of visual cortex may be protective with regard to depressive relapses (Farb, Anderson, Bloch, \& Segal, 2011). Finally, we also found that the more people tend to ruminate, the less they activate the right insula during the easy cognitive task. This is consistent with the recent findings from Mandell et al. (2014). Because the insula is implicated in self monitoring, saliency detection, and interoceptive awareness (Craig, 2009; Singer, Critchley, \& Preuschoff, 2009), we postulate that rumination tendencies may represent a maladaptive style of response with a relative lack of attention to bodily and affective signals in favor of internal cognitions (Smith \& Alloy, 2009). This fits well with a recent model of interoceptive dysregulation in depression and anxiety (Paulus \& Stein, 2010).

A major novelty of our work is to demonstrate that these correlations specifically exist when participants can let their mind wander, i.e. either at rest or when attentional resources are not fully engaged in the ongoing cognitive task. As noted in our introduction, the reciprocal relationship between rumination and cognitive control is not elucidated yet. It is unclear whether the presence of ruminations is responsible for depleting cognitive resources (bottom-up process); or instead whether impaired cognitive control might contribute to a failure to suppress rumination (top-down process) (see Mandell et al., 2014; Nejad et al., 2013). In our study, the entorhinal cortex, considered the marker of the tendency to ruminate, was generally more activated in low attentional conditions (easy > difficult task), in addition to the resting state, a pattern clearly showing such competition of the rumination process with attention. More importantly, our design could also directly establish that the differential neural activity associated with rumination is influenced by the current attentional state, and demonstrate that it is attenuated with increasing cognitive load (i.e. during the difficult task). This pattern suggests that a greater exhaustion of attentional resources by higher task demands does not exacerbate spontaneous activity related to rumination tendency, but may rather attenuate it. It remains to be determined whether this pattern, observed in non-clinical population, would hold in depressive patients. In such case, it would support the benefit of interventions introducing "more structure and constraints into activities" in order to reduce rumination in clinical populations (Watkins \& Nolen-Hoeksema, 2014).

A few behavioral studies examined the relation between attention performance and rumination in healthy people. For example, ruminators were found to exhibit more perseverative errors in the Wisconsin Card Sorting Test, but not in a simple task-switching task (Davis \& Nolen-Hoeksema, 2000). The authors concluded that rumination may interfere with performance and preferentially occur under conditions in which attention is not fully controlled by external cues. Likewise, depressed and non-depressed participants may perform similarly on a directed task but depressed patients perform worse on a free task (Watkins \& Brown, 2002). An elegant investigation of Hertel (1998) also found that, in dysphoric students, a time 
interval spent as "free" time or with "self-focused thoughts" had negative consequences on performance in a subsequent cognitive task, whereas this was not the case after a time interval with distraction by external stimuli or for non-dysphoric students (Hertel, 1998). Furthermore, in a dual-task condition, depressed patients perform worse only during high interference trials, and this cost correlates with rumination (Levens et al., 2009). Taken together, these data converge to suggest that rumination may contribute to impair the controlled allocation of cognitive resources, but that their spontaneous occurrence can be reduced by greater attentional engagement to goal-relevant information, which is consistent with the pattern found here.

Neuroimaging studies further support this functional relationship. Active task conditions may not only reduce the presence of rumination, but also reduce connectivity between anterior and posterior midline brain regions (Berman, Peltier, et al., 2011). In healthy controls, rumination or brooding correlate with difficulties in disengaging from emotionally negative material and increased activity in dorsolateral PFC, consistent with greater need for attentional control (Vanderhasselt, Kuhn, \& De Raedt, 2011). Berman, Neel, et al. (2011) also showed that depressed patients had more difficulties in expelling negative (but not positive) material from short-term memory in a direct forgetting procedure, and this difficulty correlated with rumination scores and variability of activity in left inferior frontal gyrus. However, these studies could not conclude if ruminations diminish cognitive resources, or if insufficient cognitive resources predispose to ruminations.

Here, we found that distinctive brain activation associated with rumination tendency (i.e. in entorhinal cortex) primarily occurred during low attention demands or at rest. Thus, when attention is successfully engaged by external stimuli, task-unrelated activations in memory and self-referential systems may no longer arise. Moreover, we found that accuracy in the task was negatively correlated with rumination tendencies (without any effect on RTs), suggesting that other mental processes underlying ruminations may indeed occupy attentional resources to the disadvantage of other cognitive processes under limited cognitive demand. The susceptibility to rumination may thus become more apparent during conditions of low attentional load or resting state, with higher activation of memory and self-related networks. We therefore conclude that it is not a limitation in attentional resources that predispose to ruminations, although concomitant deficits in executive control abilities and failure to inhibit self-related negative thoughts might contribute to exacerbate the ruminations (Koster et al., 2011) and perhaps engender some vicious circle. In particular, impairment in the inhibition of negative thoughts might result from hypoactivation of prefrontal cortical areas, as commonly found in depression (De Raedt \& Koster, 2010; see also Price \& Drevets, 2012).

Lastly, our study demonstrates that RRS correlates with differential (lower) activity in regions of ACC and PCC linked with the DMN in our ICA analysis of resting state. But no effect of rumination was found in these areas during the cognitive task. Thus, modulation of DMN (Greicius et al., 2007; Grimm et al., 2011) is not a general feature of rumination but might be more specific of thought processes during resting state.

\section{Limitations}

One limitation of our study is that we focused our investigation on the total RRS score, rather than specific subscores, i.e. depressive brooding and reflexive pondering, respectively thought to reflect the more negative component and the more adaptive component of rumination (Treynor, Gonzalez, \& Nolen-Hoeksema, 2003). However, as in many other recent studies, this general assessment of trait rumination appears to be a valid and sensitive method (McLaughlin \& Nolen-Hoeksema, 2011), providing a reliable marker for depressive traits and underlying neural changes (Siegle, Steinhauer, Thase, Stenger, \& Carter, 2002; Thomas et al., 2011). Another limitation is that we did not induce rumination to directly assess their impact on brain activity. However, our primary goal was to investigate the influence of cognitive states on neural processes predicting rumination trait, but not the neural underpinnings of the rumination experience itself - which are likely to implicate more widespread networks beyond medial temporal lobe. Finally, the size of our population is relatively small for establishing interindividual differences, however the consistency of our results between two different datasets provides a valuable argument in favor of these results.

\section{Conclusion}

We demonstrate that people with high scores on a questionnaire measuring the tendency to ruminate in conditions of low mood (RRS) display spontaneous activity in memory-related areas (i.e. entorhinal cortex), suggesting an increase of internally driven trains of thoughts and associations. Strikingly similar activity occurs during rest and during an ongoing cognitive task, but predominantly when the attentional task demands are low (easy condition), suggesting a competition for attentional resources between ruminations and task goals. Conversely, participants with high RRS score show reduced activation in externally driven (visual) and interoceptively driven (insula) areas, consistent with a diversion of attention to internal thoughts, away from sensory or bodily signals.

\section{Role of funding source}

This work was supported by fellowships from the Swiss National Science Foundation (Number 323500-128878) and the Foundation Vachoux to CP, as well as an award of the Geneva Academic Society (Foremane Fund) to PV.

\section{Contributors}

CP designed the study, acquired the data, analyzed the data, and drafted the article. PV designed the study, analyzed the data, and drafted the article. MD and GB designed the study, and revised the article critically for important intellectual content. YC and VS helped to analyze the data, and revised the article critically for important intellectual content. All authors contributed to and have approved the final manuscript.

\section{Conflict of interest}

All authors declare that they have no conflicts of interest.

\section{References}

Bar, M. (2009). A cognitive neuroscience hypothesis of mood and depression. Trends in Cognitive Sciences, 13(11), 456-463.

Beck, A. T., Epstein, N., Brown, G., \& Steer, R. A. (1988). An inventory for measuring clinical anxiety: Psychometric properties. Journal of Consulting and Clinical Psychology, 56(6), 893-897.

Beck, A. T., Steer, R. A., \& Brown, G. K. (1996). Manual for the Beck depression inventory (2nd ed.). San Antonio: The Psychological Corporation.

Berman, M. G., Nee, D. E., Casement, M., Kim, H. S., Deldin, P., Kross, E., et al. (2011) Neural and behavioral effects of interference resolution in depression and rumination. Cognitive, Affective, \& Behavioral Neuroscience, 11(1), 85-96.

Berman, M. G., Peltier, S., Nee, D. E., Kross, E., Deldin, P. J., \& Jonides, J. (2011). Depression, rumination and the default network. Social Cognitive and Affective Neuroscience, 6(5), 548-555.

Boettiger, C. A., \& D’Esposito, M. (2005). Frontal networks for learning and executing arbitrary stimulus-response associations. Journal of Neuroscience, 25(10), 2723-2732 
Brass, M., \& von Cramon, D. Y. (2004). Decomposing components of task preparation with functional magnetic resonance imaging. Journal of Cognitive Neuroscience, 16(4), 609-620.

Buckner, R. L. (2012). The serendipitous discovery of the brain's default network. NeuroImage, 62(2), 1137-1145.

Calhoun, V. D., Adali, T., Pearlson, G. D., \& Pekar, J. J. (2001). A method for making group inferences from functional MRI data using independent component analysis. Human Brain Mapping, 14(3), 140-151.

Chiu, Y. C., \& Yantis, S. (2009). A domain-independent source of cognitive control for task sets: Shifting spatial attention and switching categorization rules. Journal of Neuroscience, 29(12), 3930-3938.

Connolly, C. G., Wu, J., Ho, T. C., Hoeft, F., Wolkowitz, O., Eisendrath, S., et al. (2013). Resting-state functional connectivity of subgenual anterior cingulate cortex in depressed adolescents. Biological Psychiatry, 74(12), 898-907.

Cooney, R. E., Joormann, J., Eugene, F., Dennis, E. L., \& Gotlib, I. H. (2010). Neural correlates of rumination in depression. Cognitive, Affective, E' Behavioral Neuroscience. $10(4), 470-478$

Craig, A. D. (2009). How do you feel - Now? The anterior insula and human awareness. Nature Reviews Neuroscience, 10(1), 59-70.

Davis, R. N., \& Nolen-Hoeksema, S. (2000). Cognitive inflexibility among ruminators and nonruminators. Cognitive Therapy and Research, 24(6), 699-711.

De Lissnyder, E., Koster, E. H., Derakshan, N., \& De Raedt, R. (2010). The association between depressive symptoms and executive control impairment in response to emotional and non-emotional information. Cognition \& Emotion, 24(2), 264-280.

De Raedt, R., \& Koster, E. H. (2010). Understanding vulnerability for depression from a cognitive neuroscience perspective: A reappraisal of attentional factors and new conceptual framework. Cognitive, Affective, E'Behavioral Neuroscience, 10(1) $50-70$

De Vanssay-Maigne, A., Noulhiane, M., Devauchelle, A. D., Rodrigo, S., Baudoin-Chial, S., Meder, J. F., et al. (2011). Modulation of encoding and retrieval by recollection and familiarity: Mapping the medial temporal lobe networks. NeuroImage, 58(4), $1131-1138$.

Farb, N. A. S., Anderson, A. K., Bloch, R. T., \& Segal, Z. V.(2011). Mood-linked responses in medial prefrontal cortex predict relapse in patients with recurrent unipola depression. Biological Psychiatry, 70(4), 366-372.

Freton, M., Lemogne, C., Delaveau, P., Guionnet, S., Wright, E., Wiernik, E., et al (2013). The dark side of self-focus: Brain activity during self-focus in low and high brooders. Social Cognitive and Affective Neuroscience (Epub ahead of print)

Friston, K. J., Holmes, A. P., Worsley, K. J., Poline, J.-P., Frith, C. D., \& Frackowiak, R. S. J. (1994). Statistical parametric maps in functional imaging: A general linea approach. Human Brain Mapping, 2(4), 189-210.

Gotlib, I. H., \& Joormann, J. (2010). Cognition and depression: Current status and future directions. Annual Review of Clinical Psychology, 6, 285-312.

Greicius, M. D., Flores, B. H., Menon, V., Glover, G. H., Solvason, H. B., Kenna, H. et al. (2007). Resting-state functional connectivity in major depression: Abnor mally increased contributions from subgenual cingulate cortex and thalamus. Biological Psychiatry, 62(5), 429-437.

Grimm, S., Ernst, J., Boesiger, P., Schuepbach, D., Boeker, H., \& Northoff, C. (2011). Reduced negative BOLD responses in the default-mode network and increased self-focus in depression. World Journal of Biological Psychiatry, 12(8), 627-637.

Hamilton, J. P., Furman, D. J., Chang, C., Thomason, M. E., Dennis, E., \& Gotlib, I. H. (2011). Default-mode and task-positive network activity in major depressive disorder: Implications for adaptive and maladaptive rumination. Biologica Psychiatry, 70(4), 327-333.

Hertel, P.T.(1998). Relation between rumination and impaired memory in dysphoric moods. Journal of Abnormal Psychology, 107(1), 166-172.

Johnson, M. K., Nolen-Hoeksema, S., Mitchell, K. J., \& Levin, Y. (2009). Medial cortex activity, self-reflection and depression. Social Cognitive and Affective Neuroscience, 4(4), 313-327.

Joormann, J., \& D’Avanzato, C. (2010). Emotion regulation in depression: Examining the role of cognitive processes. Cognition and Emotion, 24(6), 913-939.

Kim, C., Cilles, S. E., Johnson, N. F., \& Gold, B. T. (2012). Domain general and domain preferential brain regions associated with different types of task switching: A meta-analysis. Human Brain Mapping, 33(1), 130-142.

Koster, E. H., De Lissnyder, E., Derakshan, N., \& De Raedt, R. (2011). Under standing depressive rumination from a cognitive science perspective: The impaired disengagement hypothesis. Clinical Psychology Review, 31(1), $138-145$.

Koster, E. H. W., De Lissnyder, E., \& De Raedt, R. (2013). Rumination is characterized by valence-specific impairments in switching of attention. Acta Psychologica 144(3), 563-570.

Kross, E., Davidson, M., Weber, J., \& Ochsner, K. (2009). Coping with emotions past: The neural bases of regulating affect associated with negative autobiographica memories. Biological Psychiatry, 65(5), 361-366.

Kühn, S., Vanderhasselt, M.-A., De Raedt, R., \& Gallinat, J. (2012). Why ruminators won't stop: The structural and resting state correlates of rumination and its relation to depression. Journal of Affective Disorders, 141(2-3) $352-360$

Kühn, S., Vanderhasselt, M.-A., De Raedt, R., \& Gallinat, J. (2013). The neural basis of unwanted thoughts during resting state. Social Cognitive and Affective Neuroscience (Epub ahead of print).

Levens, S. M., Muhtadie, L., \& Gotlib, I. H. (2009). Rumination and impaired resource allocation in depression. Journal of Abnormal Psychology, 118(4), 757-766.
Lundqvist, D., Flykt, A., \& Ohman, A. (1998). The karolinska directed emotional faces (CD-ROM). Stockholm, Sweden: Department of Clinical Neuroscience, Psychology Section, Karolinska Institute.

Mandell, D., Siegle, G. J., Shutt, L., Feldmiller, J., \& Thase, M. E. (2014). Neural substrates of trait ruminations in depression. Journal of Abnormal Psychology, 123(1), 35-48.

Marchetti, I., Koster, E. H. W., Sonuga-Barke, E. J., \& De Raedt, R. (2012). The default mode network and recurrent depression: A neurobiological model of cognitive risk factors. Neuropsychology Review, 22(3), 229-251.

Mayr, U., \& Keele, S. W. (2000). Changing internal constraints on action: The role of backward inhibition. Journal of Experimental Psychology, 129(1), 4-26.

McLaughlin, K. A., \& Nolen-Hoeksema, S. (2011). Rumination as a transdiagnostic factor in depression and anxiety. Behaviour Research and Therapy, 49(3), 186-193.

Meiran, N. (2010). Task-switching. In R. Hassin (Ed.), Self control in society, mind, and brain. New York: Oxford University Press.

Nejad, A. B., Fossati, P., \& Lemogne, C. (2013). Self-referential processing, rumination, and cortical midline structures in major depression. Frontiers in Human Neuroscience, 7, 666

Nolen-Hoeksema, S. (1991). Responses to depression and their effects on the duration of depressive episodes. Journal of Abnormal Psychology, 100(4), 569-582.

Nolen-Hoeksema, S. (2000). The role of rumination in depressive disorders and mixed anxiety/depressive symptoms. Journal of Abnormal Psychology, 109(3). 504-511.

Nolen-Hoeksema, S., \& Morrow, J. (1991). A prospective study of depression and posttraumatic stress symptoms after a natural disaster: The 1989 Loma Prieta earthquake. Journal of Personality and Social Psychology, 61(1), 115-121.

Nolen-Hoeksema, S., \& Watkins, E. R. (2011). A heuristic for developing transdiagnostic models of psychopathology explaining multifinality and divergent trajectories. Perspectives on Psychological Science, 6(6), 589-609.

Nolen-Hoeksema, S., Wisco, B. E., \& Lyubomirsky, S. (2008). Rethinking rumination. Perspectives on Psychological Science, 3(5), 400-424.

Paulus, M. P., \& Stein, M. B. (2010). Interoception in anxiety and depression. Brain Structure E Function, 214(5-6), 451-463.

Philipp, A. M., Weidner, R., Koch, I., \& Fink, G. R. (2012). Differential roles of inferior frontal and inferior parietal cortex in task switching: Evidence from stimulus-categorization switching and response-modality switching. Human Brain Mapping, 34(8), 1910-1920.

Philippot, P., \& Brutoux, F. (2008). Induced rumination dampens executive processes in dysphoric young adults. Journal of Behavior Therapy and Experimental Psychiatry, 39(3), 219-227.

Piguet, C., Dayer, A., Kosel, M., Desseilles, M., Vuilleumier, P., \& Bertschy, G. (2010) Phenomenology of racing and crowded thoughts in mood disorders: A theoretical reappraisal. Journal of Affective Disorders, 121(3), 189-198.

Piguet, C., Sterpenich, V., Desseilles, M., Cojan, Y., Bertschy, G., \& Vuilleumier, P. (2013). Neural substrates of cognitive switching and inhibition in a face processing task. Neurolmage, 82, 489-499.

Price, J. L., \& Drevets, W. C. (2012). Neural circuits underlying the pathophysiology of mood disorders. Trends in Cognitive Sciences, 16(1), 61-71.

Ray, R. D., Ochsner, K. N., Cooper, J. C., Robertson, E. R., Gabrieli, J. D., \& Gross, J. J. (2005). Individual differences in trait rumination and the neural systems supporting cognitive reappraisal. Cognitive, Affective, E'Behavioral Neuroscience, 5(2), $156-168$.

Schöpf, V., Windischberger, C., Kasess, C. H., Lanzenberger, R., \& Moser, E. (2010) Group ICA of resting-state data: A comparison. Magma (New York, NY), 23(5-6), 317-325.

Siegle, G. J., Steinhauer, S. R., Thase, M. E., Stenger, V. A., \& Carter, C. S. (2002). Can't shake that feeling: Event-related fMRI assessment of sustained amygdala activity in response to emotional information in depressed individuals. Biological Psychiatry, 51(9), 693-707

Singer, T., Critchley, H. D., \& Preuschoff, K. (2009). A common role of insula in feelings, empathy and uncertainty. Trends in Cognitive Sciences, 13(8), 334-340.

Smith, J. M., \& Alloy, L. B. (2009). A roadmap to rumination: A review of the definition, assessment, and conceptualization of this multifaceted construct. Clinical Psychology Review, 29(2), 116-128.

Spasojevic, J., \& Alloy, L. B. (2001). Rumination as a common mechanism relating depressive risk factors to depression. Emotion, 1(1), 25-37.

Thomas, E. J., Elliott, R., McKie, S., Arnone, D., Downey, D., Juhasz, G., et al. (2011) Interaction between a history of depression and rumination on neural response to emotional faces. Psychological Medicine, 41(9), 1845-1855.

Thomsen, D. K. (2006). The association between rumination and negative affect: A review Cognition E' Emotion, 20(8), 1216-1235.

Treynor, W., Gonzalez, R., \& Nolen-Hoeksema, S. (2003). Rumination reconsidered: A psychometric analysis. Cognitive Therapy and Research, 27(3), 247-259.

Vanderhasselt, M-A \& De Raedt, R (2012). How ruminative thinking styles lead to dysfunctional cognitions: Evidence from a mediation model. Journal of Behavior Therapy and Experimental Psychiatry, 43(3), 910-914

Vanderhasselt, M. A., Kuhn, S., \& De Raedt, R. (2011). Healthy brooders employ more attentional resources when disengaging from the negative: An event-related fMRI study. Cognitive, Affective, E Behavioral Neuroscience, 11(2), 207-216.

Watkins, E. R. (2008). Constructive and unconstructive repetitive thought. Psychol Bulletin, 134(2), 163-206.

Watkins, E., \& Baracaia, S. (2002). Rumination and social problem-solving in depression. Behaviour Research and Therapy, 40(10), 1179-1189.

Watkins, E., \& Brown, R. G. (2002). Rumination and executive function in depression: An experimental study. Journal of Neurology, Neurosurgery E Psychiatry, 72(3), 400-402. 
Watkins, E. R., \& Nolen-Hoeksema, S. (2014). A habit-goal framework of depressive rumination. Journal of Abnormal Psychology, 123(1), 24-34.

Wells, P. (2004). Depressive rumination: Nature, theory and treatment. The Atrium, Southern Gate, Chichester, England: John Wiley \& Sons Ltd.

Whitmer, A. J., \& Banich, M. T. (2007). Inhibition versus switching deficits in different forms of rumination. Psychological Science, 18(6), 546-553.

Whitmer, A. J., \& Gotlib, I. H. (2013). An attentional scope model of rumination. Psychological Bulletin, 139(5), 1036-1061.
Wiersma, J. E., van Oppen, P., van Schaik, D. J., van der Does, A. J., Beekman, A. T., \& Penninx, B. W. (2011). Psychological characteristics of chronic depression: A longitudinal cohort study. Journal of Clinical Psychiatry, 72(3), 288-294.

Zamoscik, V., Huffziger, S., Ebner-Priemer, U., Kuehner, C., \& Kirsch, P. (2014) Increased involvement of the parahippocampal gyri in a sad mood predicts future depressive symptoms. Social Cognitive and Affective Neuroscience (Epub ahead of print). 